\section{Estimating the need for dental sedation: evaluating the threshold of the IOSN tool in an adult population}

\author{
T. Liu, ${ }^{1}$ I. A. Pretty ${ }^{2}$ and M. Goodwin*2
}

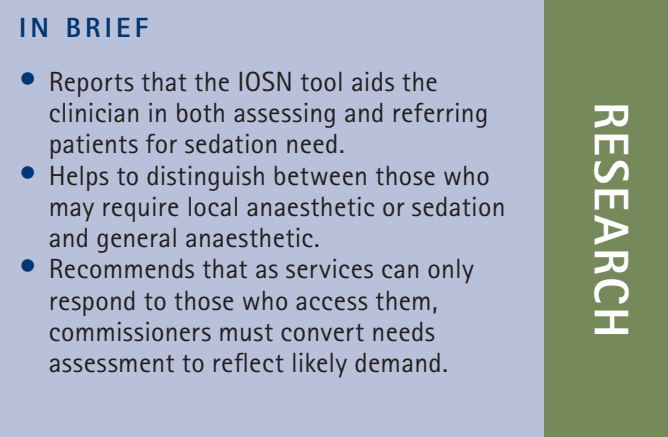

\begin{abstract}
Aim The aim of this study was, through a service evaluation, to assess the use of the IOSN tool in determining whether threshold values were appropriate for identification of IV sedation and general anaesthetic (GA) cases from a referral population. Methods A total of 105 patients were taken from a dental minor oral surgery referral service within a north west primary care trust over the course of six months. The IOSN tool was completed to assess: treatment complexity, medical and behavioural factors and patient anxiety levels. Each patient was then followed through to treatment. The type of sedation modality they received was compared to their IOSN score previously calculated and these results evaluated. Results The findings suggest that $94 \%$ of patients were treated within primary care by the MOS service, of which $58 \%$ received local anaesthetic (LA) alone and 42\% were treated by LA with IV sedation. There was a general marked trend as the IOSN score increased so did the treatment modality from LA, through sedation to GA. Logistic regression using the components of the IOSN tool to predict sedation use indicated the IOSN predictors distinguished between those who required sedation and those who didn't (chi-square $=56.411, p<0.0001, d f=3)$ with treatment complexity $(\operatorname{Exp} B=10.836, p<0.0001)$ and anxiety (Exp $B=4.319, p<0.0001$ ) shown to be significant factors in determining sedation need. Conclusions The data collected have shown that there is a positive relationship between the IOSN score and the type of treatment modality the patient received, suggesting that the threshold values are correctly set. It is concluded that IOSN tool is a useful means of aiding the clinician in both assessing and referring patients for that sedation need.
\end{abstract}

\section{INTRODUCTION}

Conscious sedation use within GDP practice has in the past been a relatively subjective process with previous evidence suggesting an inconsistency in its use, often characterised by demand rather than need. ${ }^{1}$ Some clinicians may over refer for conscious sedation, while others may never make use of a sedation service at all. Indeed, the provision of sedation services across England and Wales is far form consistent. There needs to be a clearer way to aid decision making and identify patients who require conscious sedation for dental treatment, in order to plan, commission and provide

\footnotetext{
The School of Dentistry, The University of Manchester, Higher Cambridge Street, Manchester, M15 6FH; The Dental Health Unit School of Dentistry, The University of Manchester, Williams House Manchester Science Park, Manchester, M15 6SE

*Correspondence to: Michaela Goodwin

Email: michaela.goodwin@manchester.ac.uk; Tel: 01612324711
}

\section{Online article number E22}

Refereed Paper - accepted 13 March 2013

DOI: 10.1038/sj.bdj.2013.427

${ }^{\bullet}$ British Dental Journal 2013; 214: E22 appropriate sedation services, that is, a health needs assessment (HNA) tool.

As described in the previous four papers related to this piece of research an IOSN assessment and referral tool was piloted and developed in the North West England..$^{2-5}$ The first paper described the development of a novel tool to indicate the need for conscious sedation in dentistry. ${ }^{2}$ The second paper examined the IOSN as a health needs assessment tool. The results suggested that the need for sedation did not match the number of people who actually received these services. ${ }^{3}$ However, this did not take into account the patients in the population who are non-attendees. The third paper in this series examined the potential need for sedation services among both attending and non-attending patients by assessing the reasons why people do not attend the dentist regularly. Combining the data from papers two and three together indicated that the sedation need could be $6.9 \%$ throughout the entire population. ${ }^{4}$ The fourth article focused on the use of the IOSN as a referral tool and the need for sedation in the referred patient population as determined by the IOSN score. It concluded that the IOSN tool is a useful aid in sedation referral but not a definitive verdict, as it is likely there will be a number of patients who may require sedation under exceptional circumstances. Therefore, the final judgement as to whether an individual is in need of sedation or not should be decided between both the clinician and patient. ${ }^{5}$

In this article we report on a service evaluation that recorded patients referred for minor oral surgery, the IOSN for each patient and the resulting sedation/pain relief technique used. This permitted an evaluation of the IOSN within a 'real life' setting enabling the thresholds to be further evaluated against patient outcomes.

\section{METHODS AND MATERIALS}

The study was conducted as a service evaluation, utilising adult patients referred from local dental practices, dental access centres and emergency out-of-hour services to the Minor Oral Surgery Dental Clinical 
Assessment and Treatment Service (CATS), within one particular PCT in the north west of England. This oral surgery service was developed to enable patients to receive high quality level of care and treatment without the delay and preventing unnecessary hospital attendances and long waits in the NHS. This evaluation also allowed a wide a spread of treatment complexities and patient diversity to be gained. As it was a service evaluation, no patient identifiable information was collected or assessed; that is, all responses were fully anonymised.

The data was collected over a course of six months, by one operator, in one dental CATS centre, operating one day a week. A pragmatic consecutive sample was obtained during this period, with a total of 105 patients completing the anxiety section of the IOSN form when they attend their initial consultation to CATS. The operator then completed both part one; treatment complexity, and part two; medical indicators of the IOSN form. Patient's age and gender were documented. Records of what treatment modality (either LA, IV or GA) the patient was referred for by their dentist and which of those the patient thought they were being referred for was also noted. Data were entered into the statistical software SPSS 16.0 and the IOSN scores calculated from the treatment complexity, anxiety and medical indicator scores. The patients were followed through to treatment completion and the actual treatment modality they received was recorded. Statistical analysis of the data utilised descriptives and comparisons between groups using non-parametric tests Kruskal-Wallis and Mann-Whitney U.

\section{RESULTS}

A total of 105 adult patients completed the forms and were included in the analysis. The mean age of female participants was 37.9 years old and of the males was 41.8 years old. There was a higher proportion of females compared to males (overall 61:39). Table 1 shows the female and male contributions. Of the total number of female population sampled over half (52\%) had treatment facilitated with either IV or GA whereas this was found to be just over a third (37\%) in the male population. With the resulting risk ratio of sedation use (in relation to dental treatment) in females being 1.4 times higher than in males.

Table 1 Cross tabulation between gender and treatment modality

\begin{tabular}{|l|l|l|l|l}
\hline & Outcome & & & \\
\hline Sex & LA & IV & GA & Total \\
\hline Male & 26 & 13 & 2 & $41(39 \%)$ \\
\hline Female & 31 & 29 & 4 & $64(61 \%)$ \\
\hline Total & $57(54 \%)$ & $42(40 \%)$ & $6(6 \%)$ & 105 \\
\hline
\end{tabular}

\begin{tabular}{|c|c|c|c|c|}
\hline & & Outcome & & \\
\hline & LA & IV & $\mathrm{GA}$ & Total \\
\hline IOSN Min 3-4 & 16 & 3 & 0 & 19 (18.1\%) \\
\hline Mod 5-6 & 34 & 17 & 0 & $51(48.6 \%)$ \\
\hline High 7-9 & 7 & 22 & 6 & 35 (33.3\%) \\
\hline Total & $57(54.3 \%)$ & $42(40 \%)$ & 6 (5.7\%) & 105 \\
\hline
\end{tabular}

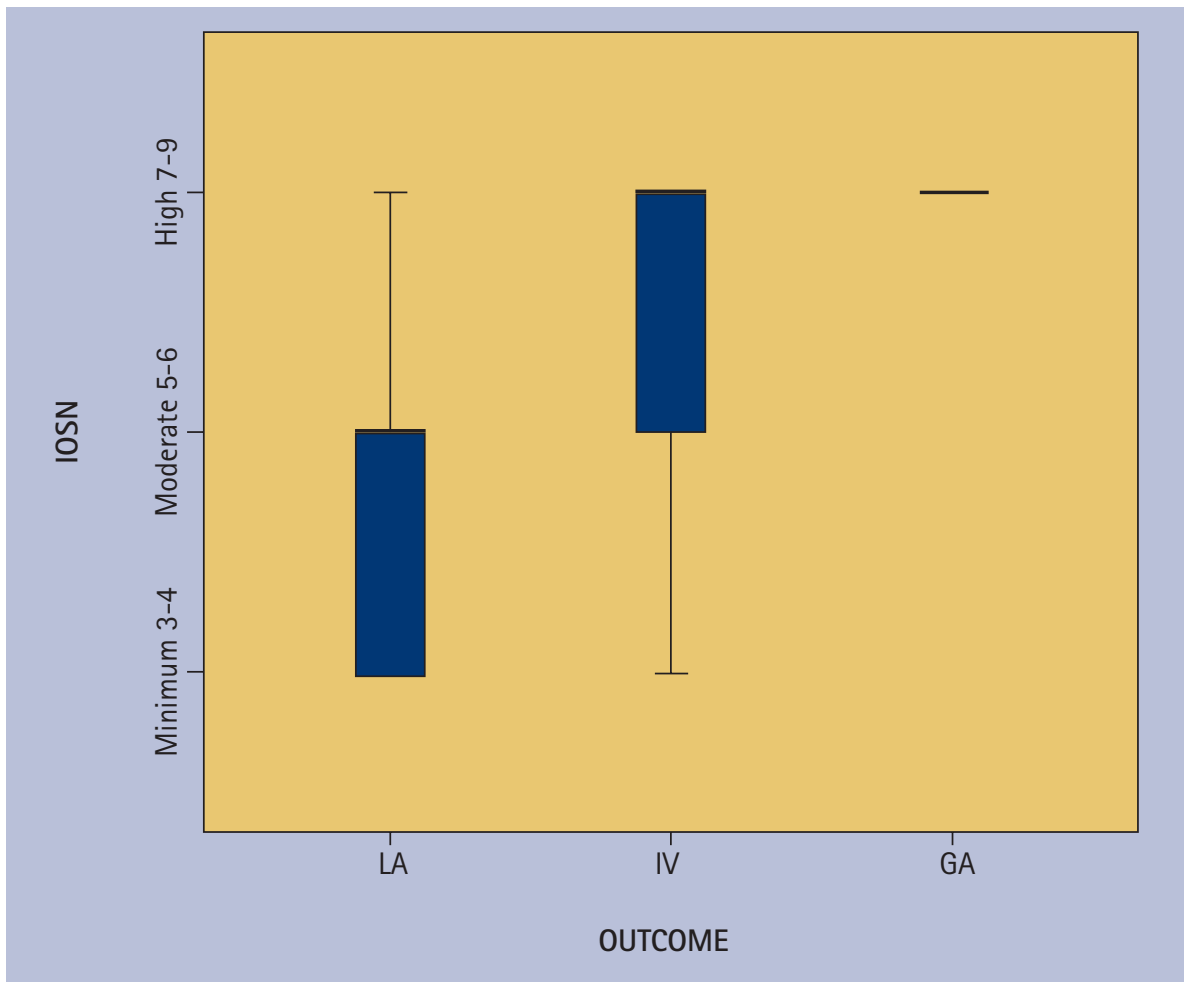

Fig 1 Relationship between IOSN score and treatment outcome

Table 2 and Figure 1 show the breakdown of the IOSN score and the treatment outcomes completed using LA, IV or GA. The IOSN scores concluded that $18.1 \%$ of patients were considered to have minimal need for sedationn and $48.6 \%$ of moderate need while $33.3 \%$ considered having high need. No patients were found within the very high need category. Within the high sedation need group 80\% of the patients were treated with either IV or GA compared to LA alone, while in the low sedation need group, only $15.8 \%$ of patients received treatment under conscious sedation. Overall Figure 1 indicates that as the IOSN scores increased, so did the sedation need that moved from LA through to GA. Differences in resulting sedation technique and IOSN score may be due to the complexity of the treatment involved or down to the patient's medical indicator, for example strong gag reflex and not as a direct reflection of their anxiety. These figures suggest that the threshold value held 


\begin{tabular}{l|l|l|}
\multicolumn{3}{|c}{ Table 3 Kruskal-Wallis test statistics } \\
\hline Outcome & N & Mean rank \\
\hline IOSN LA & 57 & 40.6 \\
\hline IV & 42 & 65.02 \\
\hline GA & 6 & 88.00 \\
\hline Total & 105 & \\
\hline H (2) $=28.653, p=0.0001$ &
\end{tabular}

\begin{tabular}{|c|c|c|c|}
\hline Outcome & $\mathrm{N}$ & Mean rank & Sum of ranks \\
\hline LA & 57 & 40.09 & 2285.00 \\
\hline IV & 42 & 63.45 & 2665.00 \\
\hline Total & 99 & & \\
\hline \multicolumn{4}{|c|}{$U=632, z=-4.388, p=0.0001$} \\
\hline IV & 42 & 23.07 & 969.00 \\
\hline GA & 6 & 34.50 & 207.00 \\
\hline Total & 48 & & \\
\hline \multicolumn{4}{|c|}{$U=66, z=-2.150, p=0.0625$} \\
\hline LA & 57 & 29.37 & 1674.00 \\
\hline GA & 6 & 57.00 & 342.00 \\
\hline Total & 63 & & \\
\hline
\end{tabular}

true for both category of patients. IOSN protocol states 'that if any patient scores four on medical indicators then they are automatically placed into a high need category (if not already categorised as such)' No participants from this study scored a four on medical history, therefore, this did not apply.

Kruskal-Wallis test was performed to determine whether there was any statistical significance between LA, IV and GA. The results showed that there was strong evidence of a difference in IOSN scores between the final treatment modalities used $\mathrm{H}(2)=28.653$ and $\mathrm{p}=0.0001$ (Table 3).

Mann-Whitney U analysis was performed between the three treatment choices: LA, IV and GA (Table 4). Due to the problem of multiple comparisons, the Bonferroni correction was used, which sets the level of significance to 0.0167 rather than $0.05(0.05 / 3)$. There was statistical significance found between LA and IV $(\mathrm{U}=632, \mathrm{z}=-4.388, \mathrm{p}=0.0001, \mathrm{r}=0.44)$ and between $\mathrm{LA}$ and $\mathrm{GA}(\mathrm{U}=21, \mathrm{z}=-3.884$,

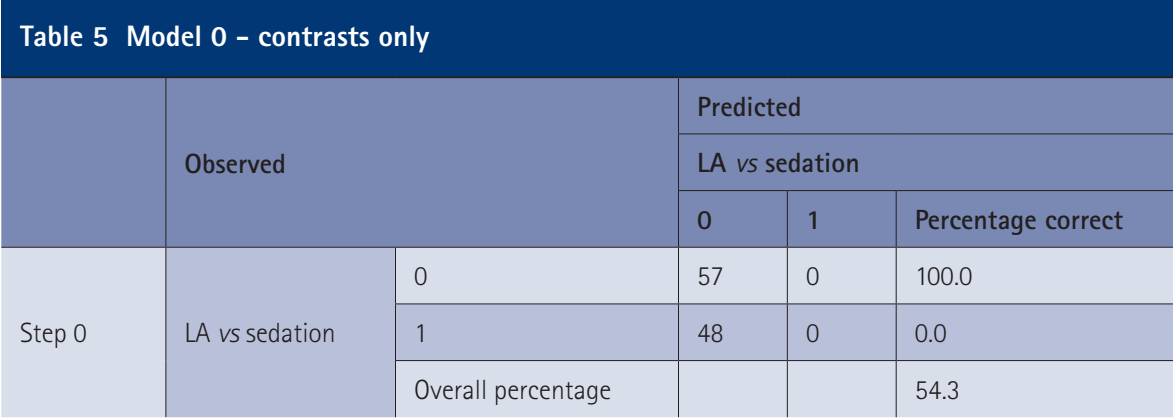

Table 6 Model 1 - IOSN components added to model

\begin{tabular}{|c|c|c|c|c|c|}
\hline & \multirow{3}{*}{\multicolumn{2}{|c|}{ Observed }} & \multicolumn{3}{|c|}{ Predicted } \\
\hline & & & \multicolumn{3}{|c|}{ LA vs sedation } \\
\hline & & & 0 & 1 & Percentage correct \\
\hline \multirow{3}{*}{ Step 1} & \multirow{3}{*}{ LA vs sedation } & 0 & 47 & 10 & 82.5 \\
\hline & & 1 & 11 & 37 & 77.1 \\
\hline & & Overall percentage & & & 80.0 \\
\hline
\end{tabular}

Table 7 Coefficient of determination

\begin{tabular}{|l|l|l|l}
\hline Step & -2 Log likelihood & Cox and Snell R Square & Nagelkerke R Square \\
\hline 1 & $88.378^{a}$ & 0.416 & 0.556 \\
\hline
\end{tabular}

Table 8 Predicted probabilities from logistic regression

\begin{tabular}{|c|c|c|c|c|c|c|c|c|c|}
\hline & & \multirow{2}{*}{ B } & \multirow{2}{*}{ S. E. } & \multirow{2}{*}{ Wald } & \multirow{2}{*}{$\mathrm{DF}$} & \multirow{2}{*}{ Sig. } & \multirow{2}{*}{$\operatorname{Exp}(B)$} & \multicolumn{2}{|c|}{$95.0 \% \mathrm{Cl}$ for $\operatorname{Exp}(\mathrm{B})$} \\
\hline & & & & & & & & Lower & Upper \\
\hline \multirow{4}{*}{ Step 1} & ANX & 1.463 & 0.306 & 22.824 & 1 & 0.000 & 4.319 & 2.370 & 7.871 \\
\hline & $\mathrm{MH}$ & -0.089 & 0.397 & 0.051 & 1 & 0.822 & 0.915 & 0.420 & 1.990 \\
\hline & $\mathrm{TC}$ & 2.383 & 0.582 & 16.749 & 1 & 0.000 & 10.836 & 3.461 & 33.920 \\
\hline & Constant & -8.427 & 1.771 & 22.630 & 1 & 0.000 & 0.000 & & \\
\hline
\end{tabular}

$\mathrm{p}=0.0001, \mathrm{r}=0.49)$ with groups showing a moderate to large effect. No significant difference was found between IV and GA $(\mathrm{U}=66, \mathrm{z}=-2.150, \mathrm{p}=0.0625, \mathrm{r}=0.31)$ when looking at the IOSN score.

Logistic regression (Tables 5-8) was performed to predict the sedation need correctly identified based on the three components of the IOSN. The number of participants who underwent treatment with LA was compared to those who received sedation (either IV or GA). A test of the constant only model (correctly identifying $54.3 \%$ of participants who would require sedation) against the full model (correctly identified $80 \%$ of participants) was statistically significant indicating the IOSN predictors distinguished between those who required sedation and those who did not (chi square $=56.411, \mathrm{p}<0.0001$ with df = 3). Nagelkerke R Square was 0.556 indicating a moderate relationship between prediction and grouping. Table 8 shows that treatment complexity (Exp $\mathrm{B}=10.836, \mathrm{p}<0.0001)$ and anxiety $(\operatorname{Exp}$ $\mathrm{B}=4.319, \mathrm{p}<0.0001$ ) were significant factors from the IOSN in determining sedation need, while the medical history $(\mathrm{MH})$ was the least significant $(\operatorname{Exp} B=0.915$, $\mathrm{p}=0.915)$.

A sensitivity and specificity test was carried out to determine the number of correct true positives and true negatives being identified. The results showed 96\% sensitivity (CI 88\% to 98\%) suggesting that it correctly identified $94 \%$ of those individuals whose IOSN score indicated they had sedation need and did indeed receive 
sedation (true positive). The $28 \%$ specificity (CI $18 \%$ to $41 \%$ ) indicated that a very low percentage of participants were correctly identified as not needing sedation and actually not receiving it (true negative). This means that of the total number of participants 39\% had a false positive type 1 error (IOSN score indicated a sedation need but no sedation received) and $3 \%$ had a false negative type 2 error (IOSN score indicated no sedation need but sedation was received).

\section{DISCUSSION}

There is evidence to suggest that some sedation services may be demand rather than needs-led. Paradoxically, some patients who are anxious about dental treatments, or patients who are undergoing complex procedures, are not being offered sedation to facilitate their treatment, therefore, there needs to be a tool to aid and support clinical decisions and to allow commissioners to understand and identify the population need in order to plan and deliver sedation services appropriately. Paper two ${ }^{3}$ of the series argued that there may be a 'postcode lottery' with local commissioning of sedation provision that can result in demand-led services in some areas while failure to meet need in others. The design of the IOSN tool assists commissioning bodies to either estimate sedation need by providing a suggestive figure that can be modified $(6.9 \%$ as a broad average), or as a tool that can be utilised to undertake their own health needs assessment. The tool can also be deployed on an individual basis as a referral management device.

Of the 105 patients who took part in the study, 61\% were females and 39\% were male, with over half of females (52\%) having treatment facilitated with either IV or GA and only just over a third (37\%) in the male population. There is a suggestion this may be a result of the general increased anxiety scores seen for females in the previous papers in this series resulting in an increased sedation need. ${ }^{3,4}$ The mean age of female participants was 37.9 years old and of the males was 41.8 years old. This corresponded to several previous studies including Nuttal et al. in $1998^{6}$ that have shown that a larger percentage of participants who are regular dental attendees were over the age of 35.
The Mann Whitney U test shows evidence of significance between the treatment outcome of LA versus IV and LA versus GA, however, no statistical significance has been reported between the IV versus GA group. This could be due to the small sample size within the GA group $(n=6)$ and hence, the lack of difference detected.

Looking at the data from the logistic regression (Table 8), this shows anxiety had the largest impact on sedation need although treatment complexity contributed a larger odds ratio (10.836). Medical history did not seem to have a significant factor towards sedation need. The fact that patient anxiety and treatment complexity appear to be the more significant factor for sedation need, leads to the questioning whether the IOSN tool could be modified and/or simplified to reflect this. Despite this, the use of the IOSN tool has shown to increase the percentage of patients correctly predicted for sedation need from $54.3 \%$ to $80 \%$. Therefore, it is beneficial to use such tools clinically to aid the assessment and referral for sedation need.

The 96\% sensitivity shows that the study was effective at determining those who have sedation need do indeed receive it, however, the low specificity at 28\% means that there is a proportion who may inappropriately receive sedation if the IOSN was relied on alone without clinical or patient input. Some factors may contribute to patients having treatment without sedation despite the indication of need. These include patients being in pain, which outweighed their anxiety to have the treatment completed under LA, patients who have moderate anxiety from the MDAS questionnaire but did not like the idea of sedation, patients that may be unable to arrange childcare or escorts required for sedation treatment, pregnant females who cannot undergo any form of sedation or simply the inability to gain adequate IV access to administer the sedation or an inability to cannulate by the dentist. The $3 \%$ type 2 errors may be explained by contribution from treatment complexity and/ or medical factors that lead to quality of patient care.

There needs to be a balance between patient 'quality' of care where an individual is offered sedation due to treatment complexity though minimal anxiety, compared to those that 'need' sedation whereby without this they would avoid dental treatment altogether. This demonstrates the importance of IOSN in not only determining those that need sedation but conversely those who do not in ensuring the service is not demand-led.

\section{CONCLUSION}

The IOSN tool is a useful means of aiding the clinician in both assessing and referring patients for that sedation need, however, it is not a definitive answer as there will always be patients who may require sedation under special circumstances and vice versa. Service providers such as CATS are set up to try and prevent a demand-led service. It must be stressed, however, that the service can only respond to those who access them. Therefore, local commissioners should convert any needs assessment to reflect the likely demand.

It should be noted that both the IOSN tool as a whole and the Modified Dental Anxiety Scale used within the tool, have been tested only on adult populations. Therefore this tool is presently only suitable for patients aged 16 years or over. It would be beneficial to investigate the use of the IOSN tool in relation to children, particularly given the development of the Modified Child Dental Anxiety Scale. ${ }^{7}$ Further research, taking into account the complex indicators that can impact on the need for paediatric sedation (or equivalent techniques) such as cooperation, maturity, behaviour or additional needs of the child should be explored.

It is clear from this evaluation that there is a positive correlation between IOSN score and the treatment outcome; with the higher the patient's IOSN score, the higher the demand for conscious sedation and general anaesthesia. The study has shown that treatment complexity and anxiety were the biggest contributors in determining sedation need for a patient. The IOSN has proven to be a helpful tool to aid their decision in the provision of sedation services across the UK. It can be expected that some patients may move between the sedation need thresholds depending on any special circumstances, such as pregnancy, severe pain or medical contra-indications that may override any pre-assessment scores for sedation need. There may also be times where non-anxious patients should 
be offered sedation to improve their overall treatment comfort for higher complex procedures. The debate arises as to whether patients should be treated by their own dentists if there is no sedation need. Often, this becomes dependent upon the experience, training and interest of the dentist in question. Careful and meticulous planning should be taken in the utilisation of the IOSN tool and it should be a final joint decision between the dentist and patient about whether there is a need for sedation with or without the need for referral.

The IOSN tool could act as a focus point when dealing with highly anxious patients and could help them overcome their dental fear by providing them with a much more pleasant experience. However, it can also be argued that for patients where treatment complexity and medical indicators are at a minimum but where anxiety is the major contributing factor to their sedation need, it may be possible to undergo some form of behavioural or psychological interventions as means to help in increasing their dental attendance.

1. Coulthard P, Horner K, Sloan P, Theaker E. Master dentistry volume 1, oral and maxillofacial surgery, radiology, pathology and oral medicine. Edinburgh: Churchill Livingstone, 2003.

2. Coulthard P, Bridgman C M, Gough L, Longman L,
Pretty I A, Jenner T. Estimating the need for dental sedation. 1. The Indicator of Sedation Need (IOSN) - a novel assessment tool. Br Dent J 2011; 211: E10.

3. Pretty I A, Goodwin M, Coulthard P et al. Estimating the need for dental sedation. 2. Using IOSN as a health needs assessment tool. Br Dent J2011; 211: E11.

4. Goodwin M, Pretty I A. Estimating the need for dental sedation. 3. Analysis of factors contributing to non-attendance for dental treatment in the general population, across 12 English primary care trusts. Br Dent J 2011; 211: 599-603.

5. Goodwin $M$, Coulthard P, Pretty | A, Bridgman C, Gough L, Sharif M O. Estimating the need for dental sedation. 4. Using IOSN as a referral tool. Br Dent J 2012; 212: E9.

6. Nuttall N M, Bradnock G, White D, Morris J, Nunn J. Dental attendance in 1998 and implications for the future. Br Dent J 2001; 190: 177-182.

7. Howard K E, Freeman R. Reliability and validity of a faces version of the Modified Child Dental Anxiety Scale. Int J Paediatr Dent 2007; 17: 281-288. 